\title{
Structure and thermal expansion mechanism of liquid InBi compound
}

\author{
I. Shtablavyi*, S. Mudry, U. Liudkevych \\ Department of Metal Physics, Ivan Franko National University of Lviv,Kyrylo i Mephodiy str. 8, 79005 Lviv, Ukraine
}

Received 10 October 2016, received in revised form 14 March 2017, accepted 16 March 2017

\begin{abstract}
The structure of the InBi compound in a liquid state in a wide temperature range has been investigated by X-ray diffraction method. As a result of analysis of temperature dependences of main structure parameters, the complicated mechanism of structural changes in the liquid state is revealed. Using the data of main structure parameters and proposed mechanism of structural transformations, the peculiarities of thermal expansion of the InBi melt have been explained.
\end{abstract}

K e y words: metallic liquids, short range order, thermal expansion, X-ray diffraction, reverse Monte-Carlo simulation

\section{Introduction}

The significant progress of micro- and nano-electronics is accompanied by investigations on searching for new lead-free solders with excellent performance properties. In a case of using heat-sensitive devices and modules, it is necessary to apply low melting point solders. Moreover, in many cases, there is a necessity for step soldering when the solder used for subsequent step has a lower melting point than that used for the preceding step [1]. That is why it is necessary to search solders with a wide range of melting points.

Considering the toxicity of classical $\mathrm{Sn}-\mathrm{Pb}$ based solders as well as poor corrosion resistance and wettability of alternative Sn-Zn solder [2], currently there are many investigations on their replacement [3]. Among the most promising Pb-free solders are the In-Bi-Sn alloys. In particular, In-31.6Bi-19.6Sn [4] and In-18.75Bi-22.15Sn [5] alloys are proposed. Besides, the Bi22-In eutectic alloy is also proposed to use for diffusion soldering of copper substrates [6]. For these alloys, as it follows from phase diagrams [7-9], the formation of InBi intermetallic phase is observed.

The InBi compound crystallizes in the tetragonal crystal system with layers of indium and bismuth, which are located in planes perpendicular to the $c$ axis
[10]. Bismuth layers are separated by indium layers between which the bonding is supposed to be weak and for that reason responsible for easy splitting $[10$, 11]. As a result of such features is a high degree of anisotropy in mutually perpendicular directions of the physical properties, measured at low and room temperatures $[12,13]$. In particular, it was found that the coefficient of thermal expansion $\alpha_{\perp}$ is positive, and $\alpha_{\|}$ is negative.

It is known that the type of atomic ordering partially can persist at a melting process. This especially concerns for compounds, in which a coordinated interatomic bonding is observed. Therefore, numerous studies of the structure of indium-bismuth alloys in solid and liquid states have been carried out [14-20]. For this system, the formation of several intermetallic compounds has been found. In earlier studies, it has been established that the structure of liquid BiIn alloys is only slightly different from that of a binary mixture with random atomic distribution [14]. It was noted that there is no evidence of a tendency towards compound formation in the liquid alloys $\mathrm{In}_{2} \mathrm{Bi}$ or InBi. Nevertheless, other studies confirmed that concentration dependences of coordination numbers and most probable interatomic distances lead to the conclusion about the fact that the Bi-In melts belong to the compound forming melts [15]. Moreover,

\footnotetext{
*Corresponding author: e-mail address: ihor.shtablavyi@lnu.edu.ua
} 
the presence of Bi-In pairs on the surface of the melt has been found [16]. It should be noted that in our previous studies of the structure and viscosity of the near-eutectic alloys it was pointed out the presence of micro-inhomogeneous atomic distribution where the chemically ordered In-Bi clusters are located in indium based matrix [17]. Availability of micro-heterogeneous structure of the In- $\mathrm{Bi}$ melts is also confirmed by the results of physical properties investigations. In particular, it was found that magnetic susceptibilities and thermoelectric power of liquid In-Bi alloys suggest the existence of a few clusters [18]. According to results of electrical conductivity investigations, it is suggested the existence of $\mathrm{Bi}$-In ordering regions [19] and the content of these regions may reach up to twenty percent for equiatomic alloy composition [20].

Another interesting, and maybe even a little strange feature of structural transformation of some metals and alloys after the melting, is reducing of interatomic distances $[21,22]$. It was revealed that for some metals, like $\mathrm{Al}$ and $\mathrm{Zn}$, interatomic distances are reduced by heating in a whole temperature interval of investigations [21]. For other metals, which are characterized by a loosely packed structure as well as covalence of interatomic bonding in the crystalline state and after the melting, the complicated nature of temperature dependence on interatomic distances is observed [23, 24]. Such structural changes are often identified with phase transitions in the liquid state [25, 26], or so-called structural transformations.

Given the facts mentioned above, a detailed study of the structure of melts and structural changes caused by temperature changes is a prerequisite for understanding the processes that occur in the formation of materials from the liquid state. In this paper, the structure of the liquid InBi compound and its structural transformations caused by temperature changes have been investigated.

\section{Experiment}

The X-ray diffraction (XRD) measurements were carried out using a high-temperature X-ray diffractometer. $\mathrm{Cu} \mathrm{K} \alpha$ radiation monochromatized with a LiF single crystal and Bragg-Brentano focusing geometry in the $2 \theta$ range from $10^{\circ}$ up to $120^{\circ}$ were used. The scattered intensity was measured with accuracy higher than $2 \%$. Intensity curves were corrected on polarization and incoherent scattering [27-29]. After the correction procedure, intensity curves were normalized to electron units by the Krogh-Moe method [30].

The thermal expansion coefficient was estimated from the data on the temperature dependence of most probable interatomic distances $r_{1}$, mean interatomic distances $\left\langle r_{1}\right\rangle, \sqrt{\left\langle r_{1}^{2}\right\rangle}$, the atomic volume $V_{\mathrm{a}}$ and the
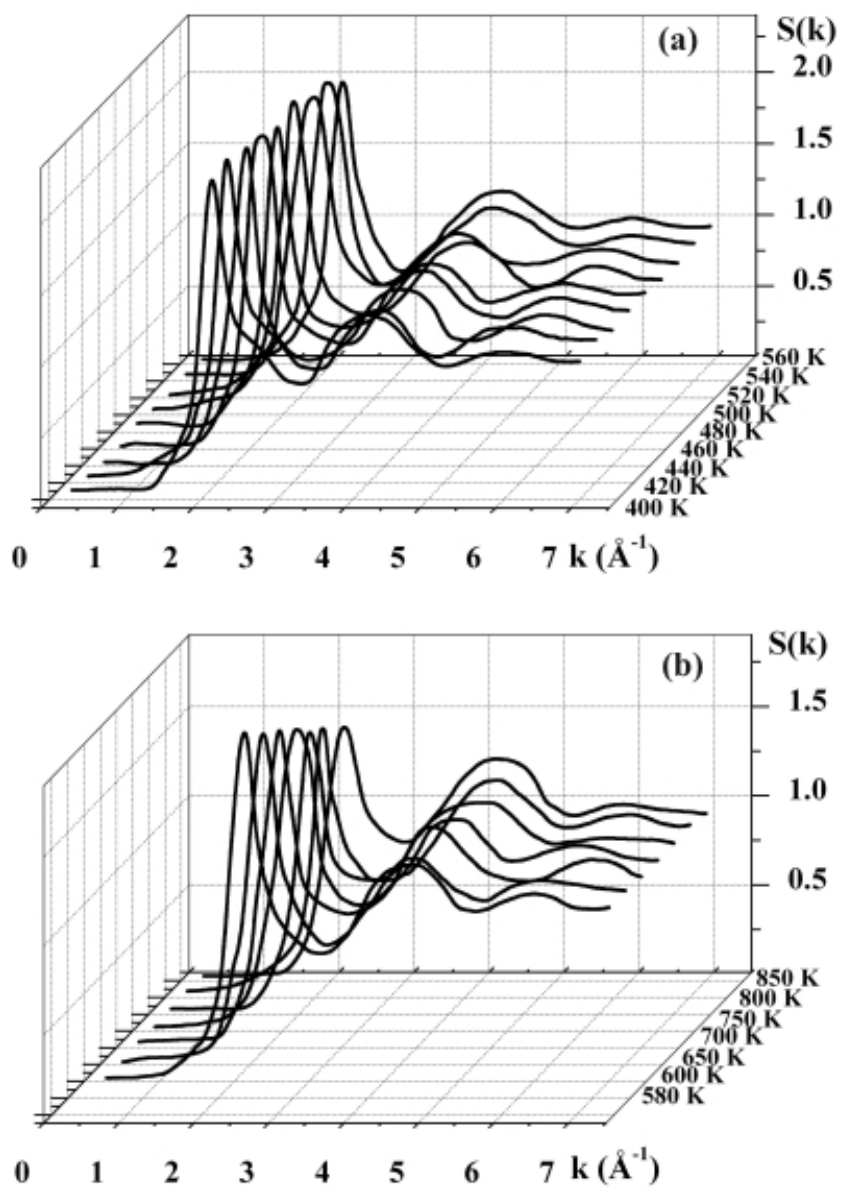

Fig. 1. Structure factors of liquid InBi.

coordination number $Z$ which were determined from pair correlation functions (PCF) and radial distribution functions (RDF) [24]. For comparison, we calculated the coefficient of thermal expansion using the data of density [31].

\section{Results and discussion}

It is known that main and direct information about the structure of the melt is commonly obtained from the analysis of structure factors (SF). Figure 1 shows the structure factors of the liquid InBi compound at different temperatures. As can be seen from the figure, the main feature of structure factors is a shoulder of the first maximum which is evident at the temperatures close to the melting point. At higher temperatures of the melt, this shoulder becomes less pronounced. The presence of the shoulder indicates the existence of atomic pairs with covalent bonding in the melt, which is typical for some group of elements, particularly for liquid bismuth. Taking into account the crystalline structure of $\mathrm{InBi}$, in which the existence of layers of bismuth atoms is observed, we can suppose 


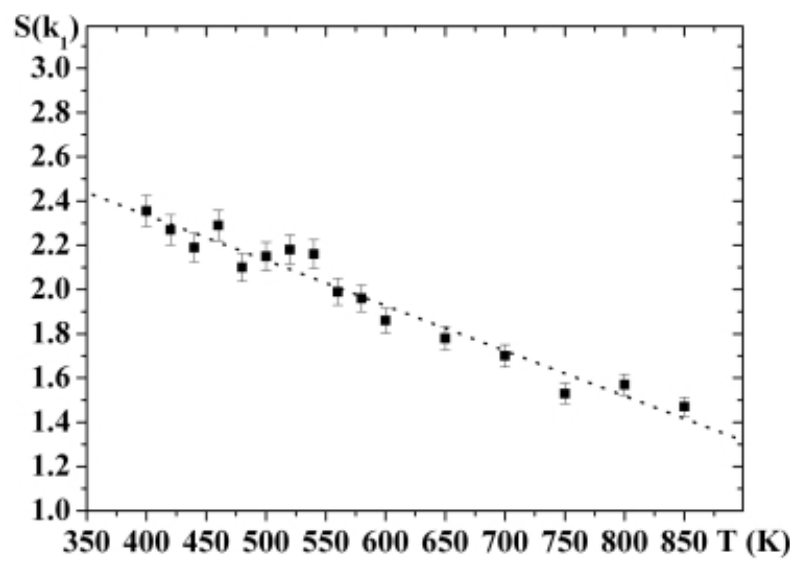

Fig. 2. Temperature dependence of the height of the SF first maximum.

that after melting some part of bismuth atoms forms clusters, randomly distributed in the In-Bi liquid matrix.

It is certain that formation of the cluster structure of the melt should be accompanied by a decrease in packing density. The packing density reduction can be confirmed by the temperature dependence of the height of the SF first maximum (Fig. 2), which, according to the hard sphere model of liquids $[29,32]$ is the most sensitive parameter, indicating such changes. As we can see in Fig. 2, the height of the first maximum decreases linearly with temperature, although at a temperature range $500-550 \mathrm{~K}$ weak deviations are observed. One of the reasons for such deviation from linearity can be the structural transformations in the liquid state in the specified range of temperatures.

Additional information on structural transformations can also be obtained from the analysis of the main structure parameters, in particular of interatomic distances and coordination numbers. However, as it can be seen from Figs. 3 and 4 the most probable interatomic distances and coordination numbers linearly decrease with increasing temperature without revealing any peculiarities in the mentioned temperature range. Only mean distances and root mean square interatomic distances show slight deviations from linearity in the vicinity of temperatures $600-650 \mathrm{~K}$ although these deviations are close to the experimental error.

The point is that the typical diffraction experiment gives only the average values of structural parameters on which we can make conclusions only on topological short-range order, which restricts the possibility of complete interpretation and deeper understanding of the structural data. More detail information about the structure of disordered systems can be obtained by the combination of diffraction experiments and computer simulations. In this work, we have used Reverse Monte Carlo (RMC) method to investigate the struc-

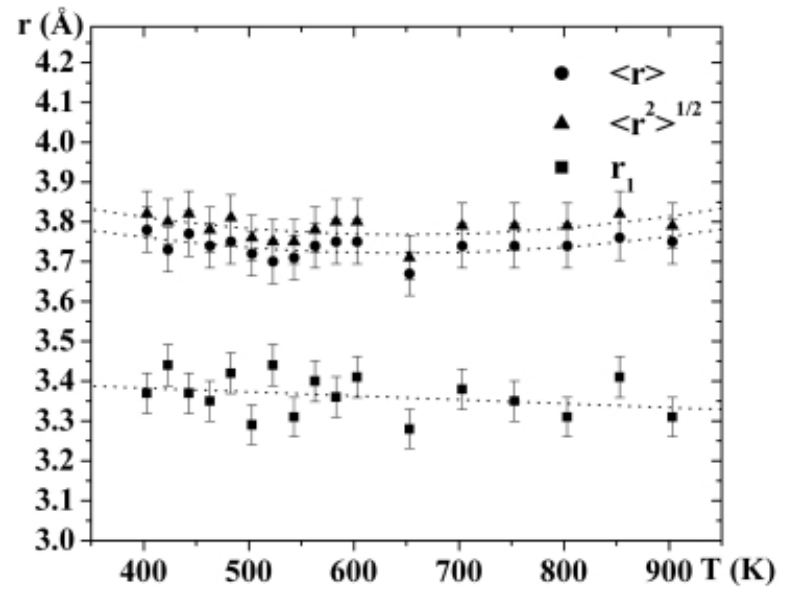

Fig. 3. Temperature dependence of the interatomic distances for InBi melt.

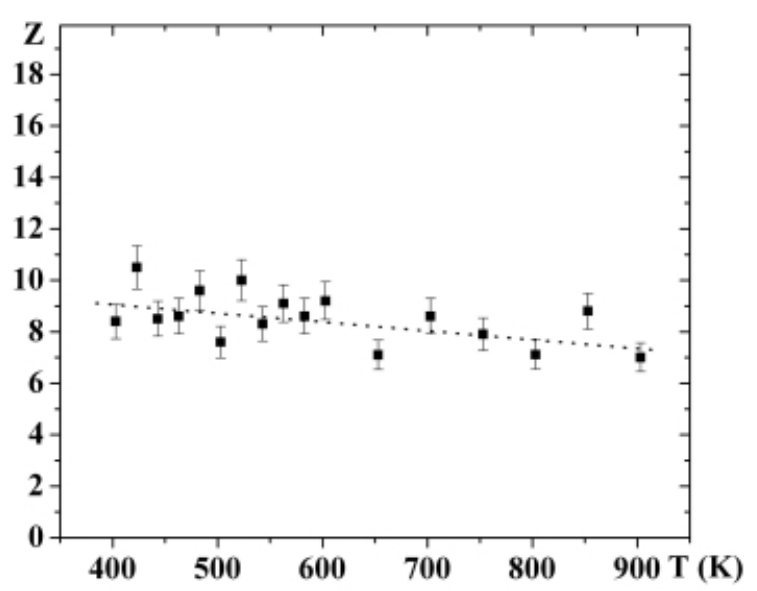

Fig. 4. Dependence of coordination number on temperature.

ture of liquid InBi compound. At modeling procedure, the primary cubic cell was arranged with 5000 atoms of stoichiometric content, which corresponded to the melt composition. The sizes of the model cube were estimated with accounting the melt density values at the temperature of the experiment. The minimum distance between atoms $r_{i j}$ was taken as the point of intersection of the left branch of the first maximum in an experimental PCF curve with the abscissa axis. RMC method allowed us to obtain the partial interatomic distances (Fig. 5) and partial coordination numbers (Fig. 6). Figure 5 also shows the temperature dependence of the partial interatomic distances in the crystalline state.

As we can see from Fig. 5, after melting $(T=383$ $\mathrm{K}$ ) interatomic distances $\mathrm{In}-\mathrm{In}$ and $\mathrm{Bi}-\mathrm{Bi}$ are reduced, while the interatomic distances In-Bi increase. Such changes of interatomic distances indicate the preferred interaction of the same kind atoms after melting and 


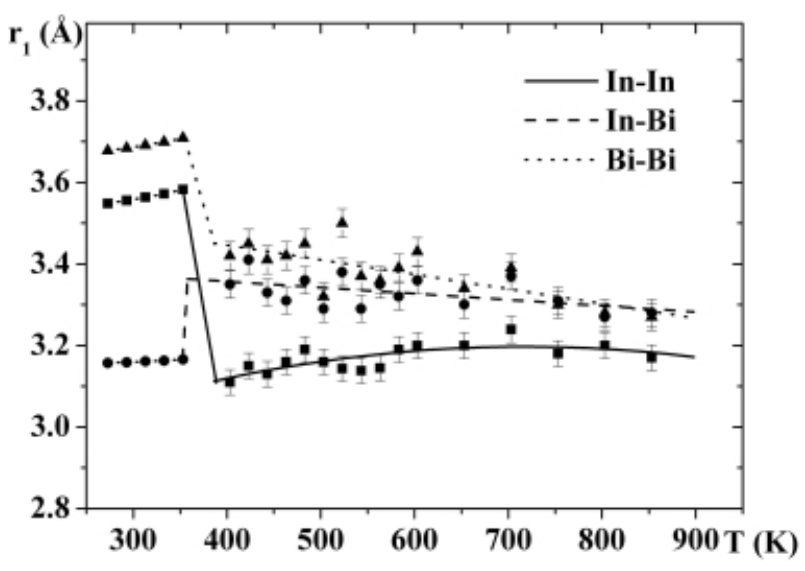

Fig. 5. Temperature dependence of partial interatomic distances for InBi melt.

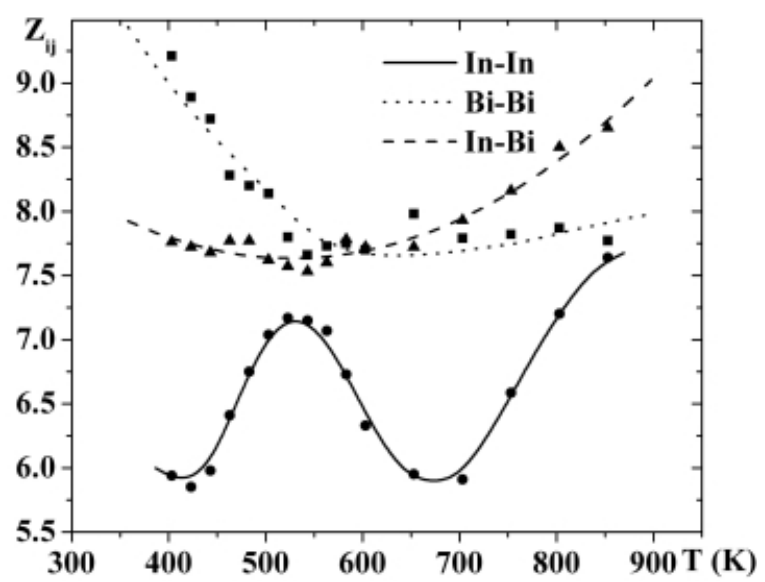

Fig. 6. Temperature dependence of partial coordination numbers for InBi melt.

confirm the formation of the Bi-based clusters, as was mentioned above.

Moreover, the reduction of the $r_{\text {In-In }}$ partial interatomic distances (Fig. 5) at the melting process and an increase of partial In-In coordination numbers just after the melting (Fig. 6), indicate the formation of indium-based clusters in which some solubility of bismuth atoms is possible.

At higher temperatures, the reduction of the In-In and $\mathrm{Bi}-\mathrm{Bi}$ partial coordination numbers is observed whereas In-Bi coordination numbers show an increase in heating within the temperature interval 525-650 $\mathrm{K}$. The mentioned temperature changes of the main structural parameters could be explained by increasing of bismuth content in indium based clusters. Further growth of temperature leads to the homogenization of the alloy.

These complex structural transformations of liquid InBi compound at heating are consistent with previously published conclusions about the thermodynamic

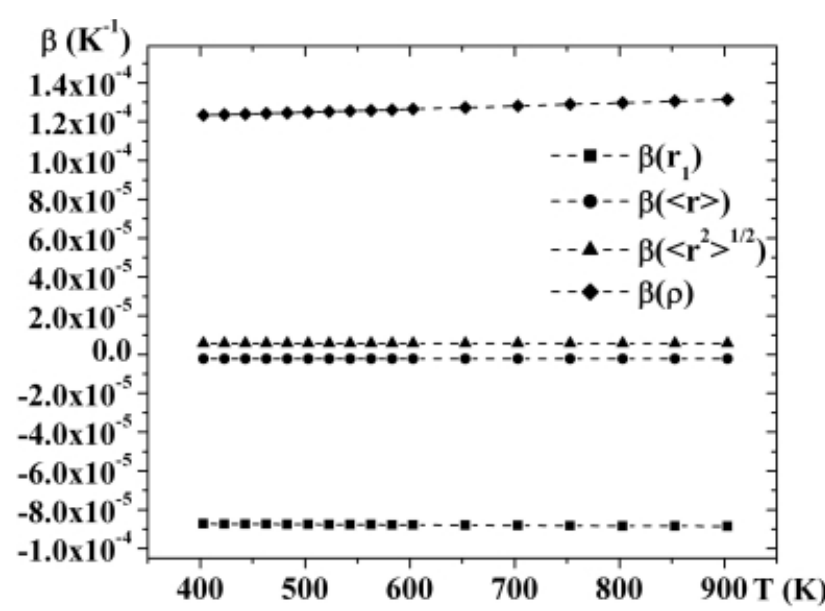

Fig. 7. Temperature dependence of calculated thermal expansion coefficient for InBi melt.

features of In-Bi alloys $[33,34]$. In particular, these studies concluded about the existence of clusters of stoichiometric composition corresponding to InBi and $\mathrm{In}_{2} \mathrm{Bi}$ compounds that coexist in the melt just upon melting. The temperature increase leads to the destruction of clusters and homogenization of the melt as it was noted in these studies.

Structural changes, accompanying melting process and the heating, affect the thermal expansion features of the liquid InBi compound. As we can see in Fig. 7, the coefficient of thermal expansion calculated based on the temperature dependence of most probable interatomic distances, which we consider as a local thermal expansion coefficient with respect to neighbor atoms [23, 24], is negative, while the values calculated based on the mean interatomic distances and mean square interatomic distances are close to zero indicating an increase in the packing density within the first coordination sphere. At the same time, the coefficient of thermal expansion calculated using the temperature dependence of the density of the melt is positive. Therefore, we can postulate predominantly about the reduction of interatomic distances after the melting, and dilation of the melt can be considered as a consequence of the increase of free volume in the melt. Our assumption is consistent with Frenkel's "hole" theory of liquids [35] and free volume theory of liquids proposed by Eyring [36] and Doolittle [37]. According to Frenkel's ideas, thermal expansion mechanism can be explained as an increase in the free volume due to "holes", without substantial changes in interatomic distances.

In this regard, using the results obtained using reverse Monte Carlo simulation, the free volume and fluctuation free volume of the liquid InBi compound at different temperatures have been calculated (Fig. 8) according to the standard methodology [38].

As can be seen in Fig. 8, the temperature depen- 


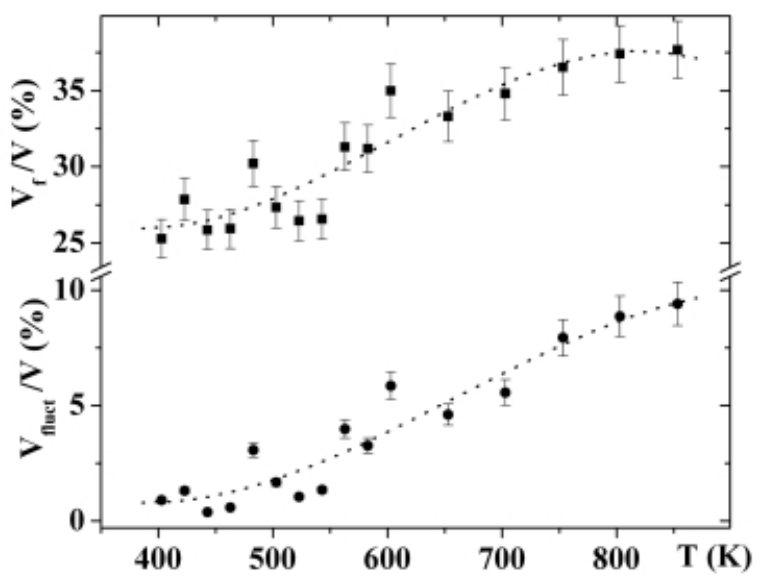

Fig. 8. Temperature dependence of free volume for InBi melt.

dences of the free volume and fluctuation free volume can be divided into three temperature subregions: 400-500 K, 500-750 K and 750-850 K, within the limits of which there is a different rate of increase of this value. These temperature intervals approximately coincide with the temperatures of structural transformations described above.

\section{Conclusions}

As a result of the investigation of the structure of liquid $\mathrm{InBi}$ compound in a wide temperature range, the mechanism of structural transformation and temperature ranges has been established. It was shown that at melting and heating up to the temperature of approximately $525 \mathrm{~K}$ the existence of clusters with preferred interaction of like kind atoms was observed. Increasing the temperature leads to complex structural transformations which result in the formation of structural units with $\mathrm{In}_{2} \mathrm{Bi}$ and $\mathrm{InBi}$ like atomic coordination is changing each other. Such structural changes result in a decrease of interatomic distances within the first coordination sphere that leads to the existence of negative local coefficient of thermal expansion. Dilation of the melt during the heating occurs mainly due to an increase in free volume. The origin of the described structural changes is supposed to be caused by the presence of the layered structure of the compound in a crystalline state.

\section{References}

[1] Mei, Z., Morris, Jr., J. W.: J. Electron. Mater., 21, 1992, p. 599. doi: $10.1007 / \mathrm{BF} 02655427$

[2] Mahmudi, R., Geranmayeh, A. R., Khanbareh, H., Jahangiri, N.: Mater. Des., 30, 2009, p. 574. $\underline{\text { doi:10.1016/i.matdes.2008.05.058 }}$
[3] Liang Zhang, Tu, K. N.: Materials Science and Engineering R Reports, 82, 2014, p. 1. doi:10.1016/j.mser.2014.06.001

[4] Efzan, M. N. E., Sharif, N. M., Yew, Ch. K., Ariga, T., Ismail, A. B., Hussain, Z: J. of Alloys and Compounds, 507, 2010, p. 290. doi:10.1016/j.jallcom.2010.07.182

[5] Huang, M. L., Zhou, Q., Zhao, N., et al.: J Mater Sci. Mater Electron., 24, 2013, p. 2624. doi:10.1007/s10854-013-1143-0

[6] Wojewoda-Budka, J., Zieba, P.: J. of Alloys and Compounds, 476, 2009, p. 164 . doi:10.1016/j.jallcom.2008.09.045

[7] Dooley, G. J., Peretti, E. A.: J. of Chemical and Engineering Data, 9, 1964, p. 90. doi:10.1021/ie60020a026

[8] Massalski, T. B.: Binary alloy phase diagram. Metals Park, OH, ASM 1990.

[9] Ohnuma, I., Cui, Y., Liu, X. Y., Inohana, Y., Ishihara, S., Ohtani, H., Kainuma, R., and Ishida, K.: J. Electron. Mater., 29, 2000, p. 1113. doi:10.1007/s11664-000-0002-y

[10] White, G. K., Collins, J. G., Scirber, J. E.: Aust. J. Phys., 43, 1990, p. 93. doi:10.1071/PH900093

[11] Jorgensen, J. O., Clark, J. B.: Phys. Rev. B, 22, 1980, p. 6149. doi:10.1103/PhysRevB.22.6149

[12] Akgos, Y. C., Farley, J. M., Saunders, G. A.: J. Phys. Chem. Sol., 34, 1973, p. 141. doi:10.1016/0022-3697(73)90071-1

[13] Fritz, I. J.: Sol. State Commun., 20, 1976, p. 299. doi:10.1016/0038-1098(76)90511-1

[14] Isherwood, S. P., Orton, B. R.: Appl. Cryst., 2, 1969, p. 219. doi:10.1107/S002188986900700X

[15] Bek, R., Nold, E., Steeb, S.: Naturforsch., 36a, 1981, p. 150. http://zfn.mpdl.mpg.de/data/Reihe_A/36/ ZNA-1981-36a-0150.pdf (in German)

[16] DiMasi, E., Tostmann, H., Shpyrko, O. G., Huber, P., Ocko, B. M., Pershan, P. S., Deutsch, M., Berman, L. E.: Physical Review Letters, 86, 2001, p. 1538. doi:10.1103/PhysRevLett.86.1538

[17] Mudry, S., Sklyarchuk, V., Yakymovych, A., Shtablavyi, I.: Physics and Chemistry of Liquids, 45, 2007, p. 675. doi:10.1080/00319100701434395

[18] Ohno, S., Tamaki, S.: Journal of the Physical Society of Japan, 38, 1975, p. 538. doi:10.1143/JPSJ.38.538

[19] Xi, Y., Zu, F.-Q., Li, X.-F., Yu, J., Liu, L.-J., Li, Q.: Physics Letters A, 329, 2004, p. 221. doi:10.1016/j.physleta.2004.06.089

[20] Geng, H., Wang, Z., Zhou, Y., Li, C.: Materials Chemistry and Physics, 133, 2012, p. 799. doi:10.1016/j.matchemphys.2012.01.096

[21] Lou, H., Wang, X., Cao, Q., Zhang, D., Zhang, J., Hu, T., Mao, H., Jiang, J.-Z.: PNAS, 110, 2013, p. 10068. doi:10.1073/pnas.1307967110

[22] Durišin, J., Saksl, K., Bednarčík, J., Michalik, Š., Gamcová, J., Pietriková, A.: Atomic Structure of Liquid Low Melting Metals. Hamburg, DESY Photon Science 2012. http://photon-science.desy.de/annual_ report/files/2012/20122234

[23] Mudry, S., Shtablavyi, I., Liudkevych, U., Winczewski, S.: Materials Science-Poland, 33, 2015, p. 767. doi:10.1515/msp-2015-0100

[24] Mudry, S., Shtablavyi, I., Liudkevych, U.: Physics and Chemistry of Liquids, 55, 2017, p. 254. doi:10.1080/00319104.2016.1198482 
[25] Wang, L., Bian, X., Liu, J.: Physics Letters A, 326, 2004, p. 429. doi:10.1016/i.physleta.2004.04.052

[26] Cheng, S.-J., Bian, X.-F., Zhang, J.-X., Qin, X.-B., Wang, Z.-H.: Materials Letters, 57, 2003, p. 4191. doi:10.1016/S0167-577X(03)00288-X

[27] Cromer, D. T., Waber, J. T.: Acta Crystallogr., 18, 1965, p. 104. doi:10.1107/S0365110X6500018X

[28] Furukawa, K.: Reports on Progress in Physics, 25, 1962, p. 395. doi:10.1088/0034-4885/25/1/310

[29] Waseda, Y.: The Structure of Non Crystalline Materials. New York, McGraw-Hill 1980.

[30] Krogh-Moe, J.: Acta Crystallogr., 9, 1956, p. 951. doi:10.1107/S0365110X56002655

[31] Berthou, P. E., Tougas, R.: Metallurgical Transactions, 1, 1970, p. 2978. doi:10.1007/BF03037849

[32] Ashcroft, N. W., Lekner, J.: Physical Rev., 145, 1966, p. 83. doi:10.1103/PhysRev.145.83
[33] Yakymovych, A., Mudry, S., Luef, Ch., Ipser, H.: Chem. Met. Alloys, 1, 2008, p. 159. http://www.chemetal-journal.org/ejournal2/ chemetal_CMA0050.pdf

[34] Kulikova, T., Mayorova, A., Shubin, A., Bykov, V., Shunyaev, K.: Kovove Mater., 53, 2015, p. 133.

[35] Frenkel, J.: Kinetic Theory of Liquids. Oxford, Clarendon Press 1946.

[36] Eyring, H.: J. Chem. Phys., 4, 1936, p. 283. doi:10.1063/1.1749836

[37] Doolittle, A. K.: Appl. Phys., 22, 1951, p. 1471. doi:10.1063/1.1699894

[38] Medvedev, N. N., Voloshin, V. P., Luchnikov, V. A., Gavrilova, M. L.: J. of Computational Chemistry, 27, 2006, p. 1676. doi:10.1002/jcc.20484 